\title{
DOENÇAS CRÔNICAS NÃO TRANSMISSÍVEIS NA \\ INFÂNCIA: REVISÃO \\ INTEGRATIVA DE \\ HIPERTENSÃO ARTERIAL \\ SISTÊMICA, DIABETES \\ MELLITUS TIPO I E \\ OBESIDADE
}

Aloísio de Freitas Jorge Júnior, Guilherme Cabral Colares, Iram Borges Moraes Rocha Filho, Lorena Souza e Silva. Doenças Crônicas não Transmissíveis na Infância: Revisão Integrativa de Hipertensão Arterial Sistêmica, Diabetes Mellitus e Obesidade. Revista Saúde Dinâmica, vol. 2, núm.2, 2020. Faculdade Dinâmica do Vale do Piranga. 


\title{
Doenças crônicas não transmissíveis na infância: revisão integrativa de hipertensão arterial sistêmica, diabetes mellitus tipo I e obesidade
}

\author{
Chronic diseases that cannot be transmissible in children: integrational review of systemic \\ arterial hypertension, type I diabetes mellitus and obesity
}

\author{
Aloísio de Freitas Jorge Júnior ${ }^{1}$, Guilherme Cabral Colares ${ }^{1}$, Iram Borges de Moraes Rocha Filho ${ }^{1}$, \\ Lorena Souza e Silva ${ }^{2}$

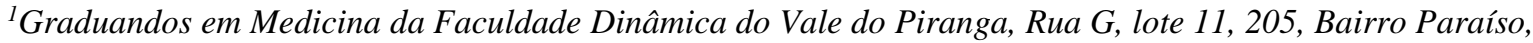 \\ Ponte Nova, $M G$ \\ ${ }^{2}$ Professora do Curso de Medicina da Faculdade Dinâmica do Vale do Piranga, Rua G, lote 11, 205, Bairro \\ Paraíso, Ponte Nova, MG. Autor correspondente:lorenanupeb@gmail.com.
}

\section{Resumo}

A terminologia "doença crônica" é utilizada na área da saúde para caracterizar qualquer morbidade incurável que afete as funções do organismo em longo prazo, solicitando assistência especial e constante. Atualmente, as Doenças Crônicas Não Transmissíveis (DCNT) são as principais causas de morte no mundo, sendo considerada um problema de saúde pública e gerando impactos negativos na vida das crianças e de seus familiares, devido as necessidades de internações, tratamentos, mudanças nos hábitos de vida e acompanhamento multiprofissional. Neste contexto, o objetivo deste trabalho foi realizar uma revisão integrativa de literatura com análise qualitativa de estudos publicados na última década sobre os principais grupos de doenças crônicas não transmissíveis na infância e seus impactos na vida dos enfermos e de seus familiares. As DCNT focadas na revisão foram a hipertensão arterial sistêmica (HAS), o diabetes mellitus tipo I (DMI) e a obesidade, que causam grandes impactos na vida das crianças, os quais podem ser revertidos por meio de intervenções amplas na promoção de saúde, para redução de seus fatores de risco, e através de melhorias na atenção à saúde, detecção precoce e tratamento oportuno, a fim de evitar complicações futuras como deficiências e incapacidades funcionais.

Palavras chave: doença crônica, obesidade, diabetes mellitus tipo 1, hipertensão, criança.

\begin{abstract}
The term chronic disease is used in the health sector to characterize any incurable morbidity that affects the organism's long-term function, needing special and constant assistance. Currently, the Chronic Non-communicable Diseases (NCDs) are the primary causes of death in the world, being considered a public healthcare problem and generating negative impacts in the lives of children and teenagers as well as their families, due to hospitalizations, treatments, life habit changes and multi-professional follow-up. In this context, the objective of this study was to perform an integrative literature revision with a qualitative analysis of studies that were published in the last decade about the main groups of chronic non-communicable childhood diseases and their impacts on the lives of the sick and of their families. The NCDs focused on the review were Systemic Arterial Hypertension (SAH), Diabetes Mellitus I (DMI) and obesity, which cause great impacts in the children's lives and that can also be reverted through a number of interventions that promote health, in order to reduce risk factors, as well as improving the primary healthcare, allowing for early diagnosis and opportune treatment in order to avoid future complications such as deficiencies and functional incapacities.
\end{abstract}

Key Words: chronic disease, obesity, diabetes mellitus type 1, hypertension, child. 


\section{INTRODUÇÃO}

A terminologia "doença crônica" é utilizada na área da saúde para caracterizar qualquer morbidade incurável que afete as funções do organismo em longo prazo, solicitando assistência especial e constante (HOLANDA, 2008). As doenças crônicas não transmissíveis (DCNT), segundo a Organização Mundial de Saúde (OMS), são um conjunto de doenças que englobam o diabetes mellitus, as doenças respiratórias crônicas, cerebrovasculares, cardiovasculares e as neoplasias, as quais compartilham os diversos fatores de riscos comportamentais e permitem a mesma abordagem nas suas prevenções (BRASIL, 2011).

Esse grupo de doenças apresentam características semelhantes, como a etiologia incerta, a presença de longos períodos de latência e curso prolongado, múltiplos fatores de risco, e poucos sintomas iniciais e por isso são frequentemente negligenciadas (BRASIL, 2011). A presença de doenças crônicas não transmissíveis na infância interferem no funcionamento do corpo, trazendo muitas dificuldades para a criança e seus familiares, pois requerem assistência contínua da equipe multidisciplinar de saúde, além de limitarem as suas atividades diárias e causar repercussões em seu processo de crescimento e desenvolvimento e impactos econômicos para as famílias, comunidades e sociedade em geral (BRASIL, 2011; HOLANDA, 2008).

Atualmente, as DCNT são as principais causas de morte no mundo, correspondendo a cerca de 72,6\% do total de óbitos em 2013, no Brasil, conforme dados do Ministério da Saúde (BRASIL, 2013). O quadro epidemiológico mundial evidência que a dinâmica nosológica tem destacado o declínio das doenças infecciosas e parasitárias nas últimas décadas, acompanhado pelo aumento das DCNT (BRASIL, 2011; PINTO; NUNES; MELLO, 2016). Aproximadamente $80 \%$ das mortes por DCNT ocorrem em países de baixa e média renda, principalmente em grupos sociais com baixa escolaridade, e um terço dessas mortes ocorre prematuramente, em pessoas com idade inferior a 60 anos (BRASIL, 2011). A maioria dessas mortes prematuras pode ser evitável com hábitos saudáveis iniciados precocemente ainda na infância, uma vez que as principais causas dessas doenças incluem fatores de risco modificáveis, como tabagismo, consumo nocivo de bebida alcoólica, inatividade física e alimentação inadequada (BRASIL, 2011).

A doença crônica altera negativamente a vida da criança devido às necessidades de internações, tratamentos, mudanças nos hábitos de vida, acompanhamento médico e, por vezes, 
necessidade de afastamento das atividades escolares. Além disso, afeta a vida dos familiares, no que diz respeito às atividades laborativas, acompanhado por grande impacto na vida econômica. Para a criança, além do impacto direto nas funções biológicas, a doença crônica não transmissível afeta as dimensões emocionais, psíquicas e sociais, carecendo de um acompanhamento multiprofissional. Além disso, é frequente o surgimento de sentimentos diversos: o medo do abandono, a interpretação da doença como um castigo, a insegurança em relação às suas possibilidades escolares, as restrições impostas pela doença, o preconceito e o bullying nas escolas. Todos esses problemas enfrentados pelas crianças e familiares, impõem modificações e formas de adaptação, processo que depende da complexidade e gravidade da doença, das estruturas e tratamentos disponíveis e do prognóstico (BRASIL, 2011; HOLANDA, 2008).

Apesar do rápido crescimento das DCNT, seu impacto pode ser revertido por meio de intervenções amplas na promoção de saúde para redução de seus fatores de risco, além de melhoria da atenção à saúde, detecção precoce e tratamento oportuno. As intervenções preventivas em doenças crônicas não transmissíveis e as estratégias de mudança de estilos de vida na população em geral, visam reduzir a prevalência dessas doenças no contexto brasileiro e mundial (BRASIL, 2011). Dentro deste contexto, é de suma importância que os profissionais da saúde tenham amplo conhecimento acerca das DCNT, sendo capaz de diagnosticar e tratar de forma rápida e efetiva, prevenindo as complicações durante a infância, como deficiências e incapacidades funcionais.

\section{MATERIAIS E MÉTODOS}

O presente estudo apresenta uma revisão integrativa de literatura com análise qualitativa sobre os principais grupos de doenças crônicas não transmissíveis na infância e seu impacto na vida dos enfermos e seus familiares. Este levantamento ocorreu durante o mês de fevereiro de 2018 e a revisão integrativa foi realizada a partir de seis etapas.

A primeira etapa consistiu na escolha da temática e a definição do objetivo: revisar literaturas atuais das doenças crônicas não transmissíveis na infância, relacionadas à hipertensão arterial sistêmica (HAS), diabetes mellitus I (DMI) e obesidade. A escolha dessas três doenças se fez devido a interrelação entre elas, onde a obesidade é apontada como um 
fator de risco comum para o desenvolvimento do DMI e HAS. Durante a segunda etapa, foi realizado um levantamento bibliográfico nas bases de dados PubMed, SciELO e ScienceDirect. A busca teve como critérios de inclusão artigos publicados na última década, na língua inglesa, portuguesa e espanhola, disponíveis on-line sob a forma de texto completo. Como técnica de investigação, foram utilizados os descritores "doença crônica", "doença na infância", "doença não transmissível" e "DCNT". Ao final deste levantamento, foram reunidos 5680 artigos.

$\mathrm{Na}$ terceira etapa foi realizada uma análise seletiva dos artigos, a partir da leitura dos títulos e resumos dos artigos. Foram excluídos os estudos que não discorriam sobre a HAS, DMI e Obesidade durante a infância, tendo sido selecionados nesta etapa 47 artigos para a leitura integral. A partir da leitura integral dos artigos selecionados na terceira etapa, houve a análise crítica dos artigos, constituindo a quarta etapa, em que foram excluídos artigos que fugiam ao objetivo proposto. Nesta etapa, observou-se que 22 artigos eram elegíveis para a realização da revisão integrativa. A Tabela 1 evidencia o quantitativo de estudos levantados e selecionados nas fases de levantamento bibliográfico e análises seletiva e crítica.

Tabela 1. Levantamento bibliográfico nas bases de dados PubMed, SciELO e ScienceDirect a partir dos descritores.

\begin{tabular}{cccc}
\hline & SciELO & PubMed & ScienceDirect \\
\hline Levantamento bibliográfico & 3149 & 82 & 2449 \\
Análise Seletiva & 19 & 0 & 28 \\
Análise Crítica & 10 & 0 & 12 \\
\hline
\end{tabular}

A Tabela 2 mostra os artigos analisados e eleitos para coleta de dados após as fases de análise seletiva e análise crítica por DCNT.

Tabela 2. Artigos elegíveis após análises seletiva e crítica.

\begin{tabular}{cccc}
\hline & Diabetes Mellitus 1 & Obesidade & Hipertensão Arterial Sistêmica \\
Análise Seletiva & 24 & 20 & 03 \\
Análise Crítica & 09 & 07 & 02 \\
\hline
\end{tabular}


A quinta etapa foi baseada na seleção de informações e agrupamento de artigos com ideias comuns e por fim, na sexta e última etapa, foi realizada a elaboração da revisão final, abrangendo a discussão das informações e evidências encontradas. Os quadros 1, 2 e 3 indicam a separação dos estudos por autor, ano de publicação e patologia analisada com breve apresentação dos principais resultados do estudo.

Quadro 1. Estudos selecionados sobre Diabetes Mellitus tipo 1.

\begin{tabular}{|c|c|c|}
\hline \multicolumn{3}{|c|}{ DIABETES MELLITUS TIPO 1} \\
\hline Autor & Ano de publicação & Principais Resultados \\
\hline Barceló e Rajpathak & 2001 & $\begin{array}{l}\text { Aumento da incidência de DM1 nos } \\
\text { últimos anos. }\end{array}$ \\
\hline Ferreira e Garcia & 2008 & $\begin{array}{c}\text { Crianças portadoras de DM1 } \\
\text { apresentam dificuldades para } \\
\text { estabelecerem relações interpessoais. }\end{array}$ \\
\hline $\begin{array}{l}\text { Greco-Soares e } \\
\text { Dell'Aglio }\end{array}$ & 2017 & $\begin{array}{l}\text { Após o diagnóstico, as crianças } \\
\text { apresentam dificuldades de aceitação, } \\
\text { mas com o tempo parecer se conformar, } \\
\text { aumentando a adesão ao tratamento. }\end{array}$ \\
\hline Jose et al. & 2009 & $\begin{array}{c}\text { Foram encontradas altas taxas de } \\
\text { glicemia na população infantil } \\
\text { analisada, necessitando abordagem } \\
\text { imediata. }\end{array}$ \\
\hline Marcelino e Carvalho & 2008 & $\begin{array}{c}\text { Crianças diagnosticadas com DM1 } \\
\text { possuem maior chance de desajustes } \\
\text { sociais por apresentarem problemas } \\
\text { emocionais como medo de morte e } \\
\text { tristeza. }\end{array}$ \\
\hline $\begin{array}{c}\text { Marques, Fornés e } \\
\text { Stringhini }\end{array}$ & 2011 & $\begin{array}{c}\text { Controle glicêmico adequado está } \\
\text { diretamente relacionado a educação, } \\
\text { boa alimentação e insulinoterapia } \\
\text { correta. }\end{array}$ \\
\hline $\begin{array}{c}\text { Ochoa, Cardoso e } \\
\text { Reyes }\end{array}$ & 2016 & $\begin{array}{c}\text { O DM1 causa grandes impactos } \\
\text { emocionais tanto na criança, quanto na } \\
\text { família. É necessário acompanhamento } \\
\text { contínuo da família e da criança. }\end{array}$ \\
\hline Okido et al. & 2017 & $\begin{array}{l}\text { Um maior tempo de diagnóstico de } \\
\text { DM1 está diretamente relacionado a } \\
\text { maior aceitação da doença pela criança. }\end{array}$ \\
\hline $\begin{array}{c}\text { Pereira, Alfenas e } \\
\text { Araújo }\end{array}$ & 2014 & $\begin{array}{l}\text { Estima-se que a falta de aleitamento } \\
\text { materno seja fator predisponente tanto } \\
\text { para DM1 quanto para DM2. }\end{array}$ \\
\hline Total & & 09 estudos \\
\hline
\end{tabular}


Quadro 2. Estudos selecionados sobre Obesidade Infantil.

\begin{tabular}{|c|c|c|}
\hline \multicolumn{3}{|c|}{ OBESIDADE } \\
\hline Autor & Ano de publicação & Principais Resultados \\
\hline Carvalho et al. & 2011 & $\begin{array}{l}\text { Obesidade infantil está ligada a má } \\
\text { alimentação e mudanças comportamentais. }\end{array}$ \\
\hline Gordia et al. & 2015 & $\begin{array}{c}\text { Os médicos avaliados demonstraram pouco } \\
\text { domínio sobre a indicação correta de } \\
\text { Atividade Física. }\end{array}$ \\
\hline Miranda et al. & 2015 & $\begin{array}{l}\text { A obesidade gera elevação dos níveis } \\
\text { séricos de ácido úrico. Altas taxas de ácido } \\
\text { úrico aumentam a resistência insulínica. }\end{array}$ \\
\hline Melzer et al. & 2015 & $\begin{array}{l}\text { Obesidade infantil está ligado ao estado } \\
\text { nutricional materno durante a gestação, } \\
\text { alimentação rica em açúcares e gorduras e } \\
\text { com predileção ao sexo feminino. }\end{array}$ \\
\hline $\begin{array}{c}\text { Paes, Marins e } \\
\text { Andreazz }\end{array}$ & 2015 & $\begin{array}{l}\text { Exercício físico, independentemente da } \\
\text { idade, mostrou-se capaz de promover } \\
\text { adaptações positivas sobre a obesidade. }\end{array}$ \\
\hline Pelegrini et al. & 2015 & $\begin{array}{l}\text { Indicadores antropométricos são boas } \\
\text { ferramentas para indicação e controle da } \\
\text { obesidade infantil. }\end{array}$ \\
\hline $\begin{array}{c}\text { Pinto, Nunes e } \\
\text { Mello }\end{array}$ & 2016 & $\begin{array}{c}\text { Aumento da prevalência de sobrepeso e } \\
\text { obesidade na população avaliada em } \\
\text { decorrência de alimentação rica em } \\
\text { açúcares. }\end{array}$ \\
\hline Total & & 07 estudos \\
\hline
\end{tabular}


Quadro 3. Estudos selecionados sobre Hipertensão Arterial Sistêmica Infantil.

\begin{tabular}{|c|c|c|}
\hline \multicolumn{3}{|c|}{ HIPERTENSÃO ARTERIAL SISTÊMICA } \\
\hline Autor & Ano de publicação & Principais Resultados \\
\hline Coelli et al. & 2011 & $\begin{array}{c}\text { Não foram demonstradas evidências da } \\
\text { associação entre HAS infantil e } \\
\text { prematuridade. Entretanto, não se pode } \\
\text { descartar tal possibilidade. }\end{array}$ \\
\hline Naghettini et al. & 2010 & $\begin{array}{c}\text { Excesso de peso e fatores hereditários } \\
\text { influenciam no desenvolvimento de HAS } \\
\text { em crianças. }\end{array}$ \\
\hline Total & & \multicolumn{2}{|c|}{ estudos } \\
\hline
\end{tabular}

\section{RESULTADOS E DISCUSSÃO}

\section{DIABETES MELLITUS TIPO 1}

De acordo com a American Diabetes Association (2015), o diabetes mellitus (DM) trata-se de um agrupamento de doenças metabólicas multifatoriais, caracterizada por hiperglicemia crônica, com alterações no metabolismo de proteínas, lipídios e carboidratos, devido à deficiência na secreção e/ou atuação da insulina (AMERICAN DIABETES ASSOCIATION, 2015; OKIDO et al., 2017). O diabetes mellitus tipo 1 (DM1) é uma das doenças crônicas não transmissíveis mais prevalentes na criança, representando cerca de 5$10 \%$ dos casos de diabetes no mundo. No Brasil, a taxa de incidência é de 7,6 a cada 100.000 em indivíduos menores que 15 anos. Segundo Duncan (2012), o DM1 caracteriza-se pela destruição autoimune das células betas pancreáticas, ficando o paciente totalmente dependente da terapia insulínica (OKIDO et al., 2017; DUCAN et al., 2012).

A DM1 é uma doença autoimune de herança multifatorial. Dessa maneira, é indicativo que há fatores externos que podem influenciar no aparecimento precoce da enfermidade. Entre esses fatores externos, Pereira et al. (2014) demonstrou que a presença de amamentação materna exclusiva até os 06 meses é um fator modificável que previne a diabetes (PEREIRA; ALFENAS; ARAÚJO, 2014). Além disso, a introdução precoce do leite de vaca na 
alimentação dos bebês pode desencadear respostas imunes, provocando a DM1 (CORREA et al., 2010).

Estudos com metodologia transversal analisaram a influência do sobrepeso na DM1 e DM2. Romualdo et al. (2014), observou o aumento da resistência insulínica em crianças obesas, concluindo que a obesidade é um fator predisponente para o desenvolvimento de distúrbios metabólicos na maturidade (ROMUALDO; NÓBREGA; ESCRIVÃO, 2014). Além disso, Miranda et al. (2015), mostraram que o aumento dos níveis séricos de ácido úrico tem correlação positiva com a desenvolvimento de resistência insulínica em crianças com sobrepeso (MIRANDA et al., 2015).

Em relação à abordagem clínica, o paciente diabético pode apresentar polifagia, poliúria, polidipsia, perda ponderal, hiperglicemia, glicosúria e acidose (JOSE et al., 2009). Segundo Rodrigues et al. (2017), geralmente, a primeira apresentação clínica é cetoacidose (28,69 \%), o que leva a investigação da doença (RODRIGUES; MOTTA, 2012; GRECOSOARES; DELLAGLIO, 2017). O diagnóstico pode ser identificado com três análises diferentes: 1) sintomas de poliúria, polidipsia e perda ponderal mais glicemia casual de > $200 \mathrm{mg} / \mathrm{dl}$; 2) glicemia de jejum $\geq 126 \mathrm{mg} / \mathrm{dl}$ (7 mmol/1); 3) glicemia de 2 horas póssobrecarga de $75 \mathrm{~g}$ de glicose $>200 \mathrm{mg} / \mathrm{dl}$ (JOSE et al., 2009).

Para medidas de controle, o exame de hemoglobina glicada (HbA1c) é o mais indicado, sendo os níveis séricos ideais de HbA1c $\leq 7,5 \%$; os níveis sub ideais ficam entre 7,6 e 9\%; e o nível de alto risco é maior que $9 \%$. Com o objetivo de controle dos índices glicêmicos, Jose et al. (2009) analisaram a HbA1c de crianças e adolescentes em três hospitais de referência de São Paulo, não sendo obtido resultados satisfatórios dos níveis de glicemia, confirmando o fato de que, apesar de todos os esforços dos profissionais da saúde, ainda há muitos desafios no controle da diabetes que precisam ser superados. A hiperglicemia crônica acarreta complicações, como: nefropatias, neuropatia e retinopatias e, por isso, é importante que o diagnóstico dessas complicações seja feito ainda na infância para melhores respostas terapêuticas (JOSE et al., 2009).

Os pacientes passam por muitas dificuldades para lidar com o diagnóstico. Em análise psicológica de 6 crianças diabéticas tipo 1 atendidas pelo Projeto DOCE (Diabetes Objetivando Controle e Educação) em Maringá, Marcelino et al. (2008) notou sentimentos de desajustes e inadequação nos contatos sociais, associados ao medo da morte e vontade de 
viver mais, além de apresentarem forte dependência da família para manterem o controle ideal da glicemia, gerando um sentimento de impotência (MARCELINO; CARVALHO, 2008). Nesse contexto, os amigos apresentam papel fundamental; embora haja afastamento nos períodos iniciais do descobrimento da doença, depois vem a reaproximação. O momento do diagnóstico é difícil tanto para o paciente quanto para amigos e familiares e o baixo nível de informação sobre a doença a priori, causa muitas incertezas (FERREIRA; GARCIA, 2008).

Além dos desafios apresentados pelas crianças, o controle adequado da glicemia depende dos responsáveis, com modificações nos hábitos de vida e medicamentos. A abordagem do cuidador familiar principal em relação às crianças portadores de DMT1 varia de acordo com o nível de escolaridade, o estresse do cuidador e a disfunção familiar, sendo fatores que influenciam na ausência de controle dos níveis glicêmicos. Em experiências relatadas pelas famílias com as crianças portadoras de DM1, conclui-se que o período de vivência com a doença associado a estruturas de ajuda, empenho e perseverança dos responsáveis potencializam o tratamento da doença crônica na infância (ZURITA-CRUZ et al., 2017).

Ademais, o diagnóstico de DM1 traz consigo impactos emocionais no ambiente familiar, pois há mudanças nos hábitos de vida de toda a família, como mudança alimentar, aumento nos gastos com alimentos e terapêuticas (OCHOA; CARDOSO; REYES, 2016). A questão financeira é uma questão importante nos desafios para o tratamento adequado, pois os custos com um indivíduo portador de DM1 chega até de 3 vezes do que uma pessoa sem a doença. Os portadores de doença crônica apresentam maior utilização dos serviços de saúde, gerando gastos absurdos com terapêuticas e controle das complicações, tornando-a um problema de saúde pública e, apesar da insulina e de matérias de consumo serem disponibilizados gratuitamente pelo Sistema Único de Saúde (SUS), nem sempre o tratamento é eficaz com os medicamentos ofertados, sendo necessário a compra de insulina análogas. Além disso, para a aquisição das insulinas de alto custo, as famílias devem recorrer ao Ministério Público, o que gera um investimento de tempo e dinheiro, sendo que as experiências de sucesso dependem do empenho das famílias, demonstrando que a rede de cuidado em saúde do Brasil é frágil e desarticulada (OKIDO et al., 2017). 
O tratamento do DM1 é constituído basicamente pela reposição insulínica, alimentação saudável e a prática de atividades físicas, juntamente com adesão ao tratamento e autocuidado, estando estes fortemente relacionados ao controle adequado da doença (MARQUES; FORNÉS; STRINGHINI, 2011). A adesão ao tratamento depende fatores socioeconômicos, da doença, da prevenção e tratamento, do emocional e/ou o sistema de saúde/equipes. De acordo com os resultados encontrados nos estudos de Okido et al. (2017), sendo utilizado um questionário para evolução da adesão ao tratamento (CEAT) com insulina, os somatórios dos resultados encontrados em crianças com DM1 variaram entre 51 e 82, com média de 70,59 (DP=5,93) (OKIDO et al., 2017). Já o autocuidado está relacionado com o acompanhamento dos pais e equipe de saúde e o tratamento tem como objetivo a prevenção de complicações agudas e crônicas, o que irá implicar em um envelhecimento com boa qualidade de vida e saúde (GRECO-SOARES; DELLAGLIO, 2017). Assim, para atingir bons resultados o profissional da saúde deve apresentar formação médica adequada, em que construa uma relação de respeito, confiança e empatia com a família (OKIDO et al., 2017).

Por fim, segundo Barceló e Rajpathak (2001) espera-se que o número de diabéticos, nas Américas, em 2025 seja de 64 milhões (RODRIGUES; MOTTA, 2012; BARCELÓ; RAJPATHAK, 2001). Dessa forma, deve-se salientar a importância do diagnóstico precoce para a prevenção de complicações futuras pois o DM1 ainda apresenta muitos desafios para o paciente, para a família e para a sociedade. Além disso, é necessário continuar os estudos entre a adesão e controle adequado da DM1, pois os dados estatísticos são fundamentais para propor medidas de controle eficazes (OKIDO et al., 2017).

\section{OBESIDADE}

A obesidade é uma enfermidade crônica definida pelo excesso de gordura corporal com posteriores malefícios à saúde (PINTO; NUNES; MELLO, 2016; PAES; MARINS; ANDREAZZ, 2015). Esse excesso de peso tornou-se um sério problema de saúde pública em todo o mundo. Estudos apresentam de forma concreta que existe uma maior probabilidade de crianças com sobrepeso serem adultos obesos (PAES; MARINS; ANDREAZZ, 2015; PELEGRINI et al., 2015; GORDIA et al., 2015; CARVALHO et al., 2011). Segundo a Organização Mundial de Saúde (OMS), a obesidade é um problema de saúde pública 
acompanhado de uma alta taxa de morbimortalidade; mais de 40 milhões de crianças com idade inferior a cinco anos apresentam sobrepeso ou obesidade com exposição a maior risco de desenvolvimento de DCNT (PINTO; NUNES; MELLO, 2016; PAES; MARINS; ANDREAZZ, 2015; CARVALHO et al., 2011).

As etiologias da obesidade estão relacionadas a diversos fatores, como: disfunções da sinalização de hormônios hipotalâmicos vinculados à saciedade, apetite e fome, polimorfismos gênicos e balanço energético positivo. Essas etiologias são potencializadas pelo desequilíbrio da elevada ingestão calórica total, em que alimentos ricos em gorduras saturadas e açúcares ultrapassam a necessidade calórica diária (PINTO; NUNES; MELLO, 2016; PAES; MARINS; ANDREAZZ, 2015; VIEIRA et al., 2017).

Da mesma forma ao que acontece em adultos, a obesidade no período infantil também está associado a complicações como: intolerância à glicose, alterações do metabolismo do colesterol, problemas ortopédicos, hipertensão arterial sistêmica, entre outros (PINTO; NUNES; MELLO, 2016; PAES; MARINS; ANDREAZZ, 2015; GORDIA et al., 2015). Além disso, fatores etiológicos também podem afetar a patogenia da obesidade, como o balanço positivo entre a ingestão calórica e o gasto energético, fatores genéticos, entre outros. Por elevar as chances de desenvolvimento da obesidade e sua posterior persistência em vida adulta, a infância vem sendo considerada como o período em que alguns autores consideram ser o mais importante da vida humana, pois o diagnóstico recente ajuda numa melhor resposta terapêutica, reduzindo assim os índices de morbimortalidade relacionados às DCNT, incluindo a obesidade infantil (PINTO; NUNES; MELLO, 2016).

Muitos métodos e modelos vêm sendo usados para a avaliação da composição corporal. Destaca-se a antropometria considerada um método simples, rápido e barato, que pode ser usado em uma grande escala de pessoas. A elevada gordura corporal tem feito com que diversos indicadores antropométricos tenham sido testados para diagnosticar os riscos que ela causa a saúde. O mais usado mundialmente é o índice de massa corporal (IMC), apesar das suas limitações (PELEGRINI et al., 2015). Contudo, outros métodos já têm sido avaliados e recomendados. A circunferência da cintura (CC), por exemplo, é um dos métodos propostos para obter os resultados mais próximos do real. A razão cintura-estatura (RCE) e o índice de conicidade (IC) também vêm sendo considerados bons indicadores para a 
avaliação e diagnóstico da gordura corporal (PELEGRINI et al., 2015; MELZER et al., 2015).

Diversos estudos têm sido realizados com o intuito de verificar o desempenho de indicadores antropométricos (IMC, CC, RCE) na avaliação e diagnóstico do excesso de gordura corporal na infância (PELEGRINI et al., 2015). Em consequência a esse excesso de peso, a concentração de gordura na região abdominal está associada à distúrbios metabólicos e risco cardiovascular, que já poderiam estar presentes no período infantil. Alguns estudos apontam que certas populações já adotam percentis e ponto de corte comum para a CC de criança. Apesar disso, ainda se tem bastantes divergências em relação aos critérios de avaliação (MELZER et al., 2015).

O exercício físico tem sido extremamente importante na prevenção e no tratamento da obesidade por promover qualidades físicas que alteram de forma positiva a composição corporal e a atividade metabólica (PAES; MARINS; ANDREAZZ, 2015; GORDIA et al., 2015). Existe uma relação inversa entre o nível de atividade física e desenvolvimento da obesidade, principalmente na infância, o que explica o apoio a essas práticas especialmente em crianças. Por outro lado, pouco se sabe sobre a atividade física para a população pediátrica, causando, assim, sua controvérsia (PAES; MARINS; ANDREAZZ, 2015). A maior parte das recomendações clínicas de tratamento e prevenção da obesidade se estabelece na união de interseções, como: mudança de hábitos alimentares, uso de medicamentos, prática regular de atividade física e outras; sendo necessário estudos da contribuição das possíveis formas e ações de tratamento (PAES; MARINS; ANDREAZZ, 2015).

O pediatra, sendo o profissional de saúde mais presente na vida das crianças e adolescentes, pode ser muito importante para a batalha contra a pandemia da obesidade infantil com enfoque na promoção, proteção, prevenção e educação para a saúde. Entretanto, o auxílio de um profissional de nutrição é essencial para que os pais conheçam como deve ser a alimentação adequada para seus filhos, bem como os tipos de alimentos que podem ser maléficos (GORDIA et al., 2015).

Há estudos que comprovam que as crianças com apoio dos pais para prática de atividades físicas são mais ativas. Sendo assim, as orientações de um educador físico, respaldadas pelo pediatra acerca da atividade física, tanto ao paciente quantos aos seus pais 
é uma estratégia propícia para uma boa aceitação deste hábito pelas crianças (GORDIA et al., 2015).

Uma melhor análise dos atuais padrões alimentares pode colaborar para a resolução da difícil relação entre saúde e dieta (VIEIRA et al., 2017). Pode-se considerar essas alterações no processo alimentar, como um dos principais motivos do desenvolvimento da obesidade infantil. $\mathrm{O}$ fator de risco mais importante para as doenças cardiovasculares na vida adulta é a obesidade, e se apresentada no período infantil, pode se expandir no futuro, indicando a necessidade de seu controle desde o início da vida, principalmente em relação aos hábitos adotados nessa etapa. Sendo assim, é de extrema importância reconhecer os padrões alimentares no período infantil em diferentes locais, potencializando, assim, o desenvolvimento de políticas públicas, ações educacionais na saúde e a nutrição no início da vida (VIEIRA et al., 2017).

\section{HIPERTENSÃO ARTERIAL SISTÊMICA (HAS)}

Segundo Coelli, et al. (2011) a hipertensão arterial do adulto se inicia na infância (COELLI et al., 2011). No entanto, a hipertensão no adulto é identificada, diagnosticada e controlada com mais facilidade que na criança; tendo em vista que o adulto já possui uma larga escala de investigação e conceitos pré-estabelecidos, como fatores cruciais sobre a doença e os parâmetros de normalidade definidos internacionalmente (NAGHETTINI et al., 2010). Com isso, o estudo da HAS na criança se torna complicado e ainda possui muitas controvérsias associadas a essa enfermidade na infância (NAGHETTINI et al., 2010, COELLI et al., 2011).

A ideia de que a HAS na infância é um exemplo de acometimento raro tem sido cada vez menos aceita, tendo em vista que alguns estudos já evidenciam um crescente aumento da hipertensão arterial na faixa etária pediátrica mesmo usando diferentes métodos e conceitos para a realização do diagnóstico (NAGHETTINI et al., 2010, COELLI et al., 2011). Nos últimos 20 anos, pesquisas e estudos feitos notaram e acompanharam a relação entre algumas enfermidades (HAS, DM, intolerância à glicose, entre outros) com a prematuridade, onde crianças nascidas com a idade gestacional inferior a 37 semanas, apresentaram ter relações precoces com tais doenças (COELLI et al., 2011). 
Em Goiânia foram avaliadas crianças entre 3 e 10 anos, moradoras dos distritos sanitários leste e sudoeste da cidade. No estudo, alguns fatores são apontados como importantes influenciadores sobre a elevação da HAS na infância, como: peso ao nascer, aleitamento materno, história familiar de hipertensão e obesidade, peso, estatura, índice de massa corporal (IMC) e pressão arterial (NAGHETTINI et al.,2010) De certa forma, fica claro que as crianças prematuras são mais sujeitas as enfermidades futuras que acompanham da sua própria condição da prematuridade, em consequência da imaturidade de órgãos e desvantagens procedentes do baixo peso ao nascer (COELLI et al., 2011)

Vale ter em vista a importância desta questão, sendo que diariamente nascem, aproximadamente, 13 milhões de prematuros, segundo a revisão publicada no Bulletin of the World Health Organization em 2010. Além disso, a principal causa de morte em crianças com idade inferior a cinco anos é a prematuridade; correspondendo com $28 \%$ da taxa de mortalidade nessa faixa etária. O Ministério da Saúde (2011) apresentou um dado que garante que a relação entre nascidos vivos prematuros e a mortalidade nessa ocasião, continua em estabilidade, com taxa média de 6,6\% (COELLI et al., 2011).

Ainda se sabe muito pouco em relação aos efeitos que a prematuridade tem sobre as doenças crônicas em períodos mais adiantes na vida. E mesmo sendo um estudo complicado e de difícil prosseguimento, muitos deles já evidenciam que existe, sim, a relação entre prematuridade e aumento de agravos crônicos. Foi na Suécia onde o estudo teve um maior número de candidatos e adeptos à pesquisa. Nele, foi possível notar que o nascimento prematuro é um fator de risco para a hipertensão arterial. Neste estudo, os homens que nasceram antes de 29 semanas de gestação possuíam o dobro de chances de terem a pressão arterial sistólica (PAS) elevada. Já os que nasceram entre 29 e 32 semanas, obtiveram 48\% de chances a mais de apresentar PAS com valores elevados. O interessante é que, mesmo depois de estabilizar os fatores familiares, como: níveis educacional, socioeconômico e idade da mãe ao nascimento; a PAS elevada ainda permaneceu. Outros estudos também já observaram essa relação. No entanto, alguns apontam pontos específicos, como já se observou valores elevados da PAS e da glicemia em jejum nas crianças prematuras quando comparadas àquelas que nasceram a termo. Em outro estudo, a associação se manteve mesmo após a adaptação para fatores relacionados a gestação e genética, posição socioeconômica e fatores da vida materna (COELLI et al., 2011). 


\section{CONCLUSÃO}

As DCNT na infância constituem um importante problema de saúde pública e podem repercutir em má qualidade de vida adulta se não abordadas de forma efetiva e em tempo hábil. Diante disso, o presente estudo buscou elucidar os principais fatores de risco para obesidade, diabetes mellitus tipo 1 e hipertensão arterial sistêmica, bem como medidas essenciais para prevenir e tratar estas condições.

O tema abordado é de reponsabilidade multiprofissional. Nesse contexto, o estudo contribui para a atualização dos diferentes profissionais da saúde, capacitando-os para o reconhecimento e abordagem das doenças crônicas não transmissíveis mais prevalentes de uma fase muito importante da vida humana - a infância.

\section{REFERÊNCIAS}

AMERICAN DIABETES ASSOCIATION (Oregon). Diabetes Care. Portland, v. 38, (Suppl 1), S70-6, 2015.

BARCELÓ, Alberto; RAJPATHAK, Swapnil. Incidence and prevalence of diabetes mellitus in the Americas. Revista Panamericana de Salud Pública, Washington, v. 10, n. 5, p. 300308, 2001.

BRASIL. Ministério da Saúde. Secretaria de Vigilância em Saúde. Departamento de Análise de Situação de Saúde. Plano de ações estratégicas para o enfrentamento das doenças crônicas não transmissíveis (DCNT) no Brasil 2011-2022. Brasília, DF: Ministério da Saúde, 2011. 160 p.

BRASIL. INSTITUTO BRASILEIRO DE GEOGRAFIA E ESTATÍSTICA. Percepção do estado de saúde, estilos de vida e doenças crônicas. Pesquisa Nacional de Saúde (PNS). Rio de Janeiro, 2013. 180p.

CARVALHO, Maria Ana et al. Análise comparativa de métodos de abordagem da obesidade infantil. Revista Portuguesa de Saúde Pública, v. 29, n. 2, p. 148-156, 2011.

COELLI, Anna Paula et al. Prematuridade como fator de risco para pressão arterial elevada em crianças: uma revisão sistemática. Revista Cadernos de Saúde Pública, v. 27, n. 2, p. 207-218, 2011.

CORREA, Fernanda Ferreira et al. Open challenge for the diagnosis of cow's milk protein allergy. Jornal de Pediatria. v. 86, n. 2, p. 163-166, 2010. 
DUCAN, Bruce Bartholow et al. Chronic non-communicable diseases in Brazil: priorities for disease management and research. Revista de Saúde Pública, v. 46, p. 126-134, 2012. Supl. 1.

FERREIRA, Bruno Eduardo Silva; GARCIA, Agnaldo. Aspectos da amizade de adolescentes portadores de diabetes e câncer. Revista Estudos em Psicologia, v. 25, n. 2, p. 293-300, 2008.

GORDIA, Alex Pinheiro et al. Conhecimento de pediatras sobre a atividade física na infância e adolescência. Revista Paulista de Pediatria, v. 33, n. 4, p. 400-406, 2015.

GRECO-SOARES, Juliana Prytula; DELL'AGLIO, Débora Dalbosco. Adesão ao tratamento em adolescentes com diabetes mellitus tipo 1. Revista Psicologia, Saúde e Doenças, v. 18, n. 2, p. 322-334, 2017.

HOLANDA, Eliane Rolim. Doença crônica na infância e o desafio do processo de escolarização: percepção da família. 2008. 116 f. Dissertação (Mestrado em Enfermagem) Universidade Federal da Paraíba, João Pessoa.

JOSE, Laura Pereira da Silva et al. Clinical and laboratory profile of pediatric and adolescent patients with type 1 diabetes. Jornal de Pediatria, v. 85, n.6, p. 490-494, 2009.

MARCELINO, Daniela Botti; CARVALHO, Maria Dalva de Barros. Aspectos emocionais de crianças diabéticas: experiência de atendimento em grupo. Revista Psicologia em Saúde, v. 13, n. 2, p. 345-350, 2008.

MARQUES, Rosana de Morais Borges; FORNÉS, Nélida Schmid; STRINGHINI, Maria Luiza Ferreira. Fatores socioeconômicos, demográficos, nutricionais e de atividade física no controle glicêmico de adolescentes portadores de diabetes melito tipo 1. Arquivo Brasileiro de Endocrinologia e Metabologia, v. 55, n. 3, p. 194-202, 2011.

MIRANDA, Josiane Aparecida de et al. O papel do ácido úrico na resistência insulínica em crianças e adolescentes com obesidade. Revista Paulista de Pediatria, v. 33, n. 4, p. 431436, 2015.

MELZER, Matheus Ribeiro Theodósio Fernandes et al. Fatores associados ao acúmulo de gordura abdominal em crianças. Revista Paulista de Pediatria, v. 33, n. 4, p. 437-444, 2015.

NAGHETTINI, Alessandra Vitorino et al. Avaliação dos Fatores de Risco e Proteção Associados à Elevação da Pressão Arterial em Crianças. Arquivos Brasileiros de Cardiologia, v. 94, n. 04, p. 486-491, 2010.

OCHOA, M.; CARDOSO, M.; REYES, V. Emociones de la familia ante el diagnóstico de diabetes mellitus tipo 1 en el infante. Enfermería Universitaria, v. 13, n. 1, p. 40-46, 2016. 
OKIDO, Aline Cristiane Cavicchioli et al. Care demands of children with type 1 Diabetes Mellitus. Escola Anna Nery Revista de Enfermagem, Rio de Janeiro, v. 21, n. 2, p.1-7, 2017.

PAES, Santiago Tavares; MARINS, João Carlos Bouzas; ANDREAZZ, Ana Eliza. Efeitos metabólicos do exercício físico na obesidade infantil: uma visão atual. Revista Paulista de Pediatria, v. 33, p.122-129, 2015

PELEGRINI, Andreia et al. Indicadores antropométricos de obesidade na predição de gordura corporal elevada em adolescentes. Revista Paulista de Pediatria, v. 33, n. 1, p.56-62, 2015.

PEREIRA, Patrícia Feliciano; ALFENAS, Rita de Cássia G.; ARAÚJO, Raquel Maria A. Does breastfeeding influence the risk of developing diabetes mellitus in children? A review of current evidence. Jornal de Pediatria, Rio de Janeiro, v. 90, n. 1, p. 7-15, 2014.

PINTO, Renata Paulino; NUNES, Altacílio Aparecido; MELLO, Luane Marques de. Análise dos fatores associados ao excesso de peso em escolares. Revista Paulista de Pediatria, v. 34, n. 4, p. 460-468, 2016.

RODRIGUES, Mônica Loureiro Celino; MOTTA, Maria Eugênia Farias Almeida. Mechanisms and factors associated with gastrointestinal symptoms in patients with diabetes mellitus. Jornal de Pediatria, v. 88, n. 01, p. 17-24, 2012.

ROMUALDO, Monica Cristina dos Santos; NÓBREGA, Fernando José de; ESCRIVÃO, Maria Arlete Meil Schimith. Insulin resistance in obese children and adolescents. Jornal de Pediatria, v. 90, n. 6, p. 600-607, 2014.

VIEIRA, Diva Aliete dos Santos et al. Nutritional quality of dietary patterns of children: are there differences inside and outside school? Jornal de Pediatria, v. 93, n. 1, p. 47-57, 2017.

ZURITA-CRUZ, Jessie Nallely et al. Influence of the informal primary caretaker on glycemic control among prepubertal pediatric patients with type 1 diabetes mellitus. Jornal de

Pediatria, v 93, n. 2, p. 136-141, 2017.

Declaração de Interesse

Os autores declaram não haver nenhum conflito de interesse

\section{Financiamento}

Financiamento próprio

\section{Colaboração entre autores}

Aloísio de Freitas Jorge Júnior, Guilherme Cabral Colares e Iram Borges Moraes Rocha Filho: Conceberam o estudo, contribuíram para o seu design, analisaram e interpretaram os dados, bem como escreveram o manuscrito.

Lorena Souza e Silva: contribuiu no conteúdo intelectual do manuscrito, no desenho e interpretação dos dados e revisão da escrita do manuscrito. 